\title{
gु \\ Correlated bosons on a lattice: Dynamical mean-field theory for Bose-Einstein condensed and normal phases
}

\author{
Krzysztof Byczuk ${ }^{1,2}$ and Dieter Vollhardt ${ }^{1}$ \\ ${ }^{1}$ Theoretical Physics III, Center for Electronic Correlations and Magnetism, Institute of Physics, \\ University of Augsburg, 86135 Augsburg, Germany \\ ${ }^{2}$ Institute of Theoretical Physics, University of Warsaw, ulica Hoża 69, 00-681 Warszawa, Poland
}

(Received 11 March 2008; published 10 June 2008)

\begin{abstract}
We formulate a bosonic dynamical mean-field theory (B-DMFT) which provides a comprehensive, thermodynamically consistent framework for the theoretical investigation of correlated lattice bosons. The B-DMFT is applicable for arbitrary values of the coupling parameters and temperature and becomes exact in the limit of high spatial dimensions $d$ or coordination number $Z$ of the lattice. In contrast to its fermionic counterpart, the construction of the B-DMFT requires different scalings of the hopping amplitudes with $Z$ depending on whether the bosons are in their normal state or in the Bose-Einstein condensate (BEC). A detailed discussion of how this conceptual problem can be overcome by performing the scaling in the action rather than in the Hamiltonian itself is presented. The B-DMFT treats normal and condensed bosons on equal footing and thus includes the effects caused by their dynamic coupling. It reproduces all previously investigated limits in parameter space, such as the Beliaev-Popov and Hartree-Fock-Bogoliubov approximations, and generalizes the existing mean-field theories of interacting bosons. The self-consistency equations of the B-DMFT are those of a bosonic single-impurity coupled to two reservoirs corresponding to bosons in the condensate and in the normal state, respectively. We employ the B-DMFT to solve a model of itinerant and localized, interacting bosons analytically. The local correlations are found to enhance the condensate density and the BEC transition temperature $T_{\mathrm{BEC}}$. This effect may be used experimentally to increase $T_{\mathrm{BEC}}$ of bosonic atoms in optical lattices.
\end{abstract}

DOI: 10.1103/PhysRevB.77.235106

PACS number(s): 71.10.Fd, 67.85.Hj

\section{INTRODUCTION}

The observation of the Bose-Einstein condensation (BEC) in ultracold, atomic gases has greatly stimulated research into the properties of this fascinating quantum state of matter. ${ }^{1}$ In particular, experiments with alkali atoms confined in optical lattices ${ }^{2-4}$ have renewed the theoretical interest ${ }^{5-8}$ in the physics of strongly correlated bosons on lattices, which promises significant new insights and even applications in fields such as quantum computing. ${ }^{9}$

The investigation of correlated lattice bosons is not only relevant for ultracold bosonic atoms in optical lattices but has a long history starting with the formulation of Matsubara and Matsuda ${ }^{10,11}$ and Morita ${ }^{12}$ of a lattice model of liquid ${ }^{4} \mathrm{He}$. A new direction of research was initiated by Fisher et al. ${ }^{13}$ who studied lattice bosons with and without disorder to explore the superfluid-insulator transition ${ }^{14-18}$ and boson localization observed in ${ }^{4} \mathrm{He}$ absorbed in porous media. ${ }^{19}$ Granular superconductors forming the weak Josephson junctions have also been described by interacting lattice bosons. ${ }^{20,21}$ Recently, quantum phase transitions in magnetic systems such as $\mathrm{TlCuCl}_{3},{ }^{22}$ which can be induced by tuning the magnetic field, have been interpreted as the BEC of magnons. ${ }^{23}$ Bosonic supersolids ${ }^{24,25}$ promise to be yet another fascinating state of bosonic matter.

In this paper, we formulate a comprehensive, thermodynamically consistent theory of correlated lattice boson systems, namely, a bosonic dynamical mean-field theory (BDMFT) which is applicable for arbitrary values of the coupling parameters and temperature. The B-DMFT includes all local, dynamical correlations of the many-boson system and becomes exact in the limit of infinite space dimensions in analogy with its successful fermionic counterpart. ${ }^{26-29}$ With the B-DMFT, we are able to solve a lattice model of itinerant and localized, interacting bosons. The local correlations lead to an enhancement both in the BEC transition temperature $T_{\mathrm{BEC}}$ and the condensate fraction as compared to the noninteracting system. Hence, bosonic correlations can be employed in the laboratory to reach higher values of $T_{\mathrm{BEC}}$.

This paper is organized as follows. In Sec. II, we introduce the bosonic Hubbard model and explain the specific problems arising in the construction of a B-DMFT in the limit of large coordination number $Z$ of the lattice, namely, the problem of how to scale the hopping amplitudes with $Z$. The self-consistency equations and the general structure of the B-DMFT are discussed in Sec. III. The comprehensive nature of the B-DMFT is demonstrated in Sec. IV by explicitly reproducing results previously obtained in special limits of parameter space and by deriving other bosonic mean-field theories. In Sec. V, the B-DMFT is employed to solve a bosonic version of the Falicov-Kimball model and it is shown that correlation effects lead to an enhancement of $T_{\mathrm{BEC}}$. Conclusions and an outlook in Sec. VI close the presentation.

\section{CORRELATED LATTICE BOSONS}

\section{A. Generalized bosonic Hubbard model}

In the following, we consider a many-particle system with different species of bosons as can be realized in optical lattices. ${ }^{3,4}$ This may either involve different atoms as, for example, in a binary mixture of ${ }^{87} \mathrm{Rb}$ and ${ }^{7} \mathrm{Li}$, or one type of atom in different hyperfine quantum states such as ${ }^{87} \mathrm{Rb}$, 
where the total nuclear spin $I=3 / 2$ adds to the spin $S=1 / 2$ of the valence $s$ electron, giving states with $F=I+S=1$ or 2 . Such systems may be modeled by a generalized bosonic Hubbard Hamiltonian $5,10,13,30$

$$
H=\sum_{i j \nu} t_{i j}^{\nu} b_{i \nu}^{\dagger} b_{j \nu}+\frac{1}{2} \sum_{i \mu \nu} U_{\mu \nu} n_{i \mu}\left(n_{i \nu}-\delta_{\mu \nu}\right) \equiv H_{0}+H_{\mathrm{int}},
$$

where $n_{i \nu}=b_{i \nu}^{\dagger} b_{i \nu}$ is the occupation number operator for bosons of species $\nu$. Furthermore, $t_{i j}^{\nu}$ are hopping amplitudes of $\nu$ bosons and $U_{\mu \nu}$ are local density-density interactions between $\mu$ and $\nu$ bosons on the same lattice site. An exchange interaction for spinor bosons can be easily included. In general, the many-boson model [Eq. (1)] in unsolvable.

\section{B. Construction of a comprehensive mean-field theory}

The explanation of experiments with correlated lattice bosons in quantum optics and condensed-matter physics requires a comprehensive theoretical scheme for the investigation of Hamiltonian (1). In particular, it must be capable of describing thermal and quantum phase transitions and thus provide the phase diagram and the thermodynamics for the entire range of microscopic parameters. In the case of lattice fermions, such a framework already exists: the dynamical mean-field theory (DMFT). ${ }^{28}$ Indeed, the DMFT has proved to be a very successful, comprehensive mean-field theory for models and materials with strongly correlated electrons. ${ }^{29,31}$ In particular, it provides a quantitative description of the Mott-Hubbard metal-insulator transition, photoemission spectra, magnetic phases, and other correlation induced phenomena. The DMFT has the virtue of becoming exact in the limit of infinite space dimensions $d$ or, equivalently, infinite coordination number $Z$, i.e., number of nearest neighbors $\left(Z=2 d\right.$ for a $d$-dimensional hypercubic lattice). ${ }^{26}$ This limit is well known to produce mean-field theories which are diagrammatically controlled and whose free energies have no unphysical singularities (e.g., the Weiss mean-field theory for the Ising or Heisenberg spin models). ${ }^{32}$ To obtain a physically meaningful mean-field theory, the free energy of the model has to remain finite in the limit $d$ or $Z \rightarrow \infty .{ }^{26}$ This requires a suitable scaling of the coupling parameters with $d$ or $Z$, e.g., $J \rightarrow \widetilde{J} / Z$, where $\widetilde{J}=$ const, for Ising spins with nearest-neighbor coupling $J$. While for the Ising model the scaling is self-evident, this is not so for more complicated models. Namely, fermionic or bosonic many-particle systems are usually described by a Hamiltonian consisting of several noncommuting terms, each of which is associated with a coupling parameter, e.g., a hopping amplitude or interaction. In such a case, the question of how to scale these parameters has no unique answer since this depends on the physical effects one wishes to explore. ${ }^{33,34}$ In any case, the scaling should be performed such that the model remains nontrivial and its free energy stays finite in the $Z \rightarrow \infty$ limit. By "nontrivial," we mean that not only $\left\langle H_{0}\right\rangle$ and $\left\langle H_{\text {int }}\right\rangle$ but also the competition between these terms as expressed by $\left\langle\left[H_{0}, H_{\text {int }}\right]\right\rangle$ should remain finite; here, $\langle\cdots\rangle$ denotes the quantum and statistical average of operators. In the literature on lattice bosons, the $d \rightarrow \infty$ limit was so far considered only in connection with the distance-independent ("infinite-range") hopping of the bosons ${ }^{15,17,18}$ in which the mean-field theory of Fisher et al. ${ }^{13}$ for the Bose-condensed phase becomes exact. As will be discussed below, this is a static mean-field theory since normal and condensed bosons are not dynamically coupled. In particular, in the normal phase, one has $\left\langle\left[H_{0}, H_{\text {int }}\right]\right\rangle=0$ and the lattice problem is reduced to a singlesite ("atomic") problem where particles are immobile. Another static mean-field theory is the Bogoliubov approximation, which yields a good weak-coupling mean-field theory for bosons in a continuum. For lattice bosons, this approximation fails to describe the Mott superfluid-insulator transition.

Evidently, a dynamical mean-field theory is needed to describe the rich physics of interacting lattice bosons, e.g., cold atoms in optical lattices, ${ }^{2}$ within one conceptual framework. A comprehensive DMFT for correlated lattice bosons, i.e., a theory which can describe normal and condensed bosons on the same footing, did not exist up to now. In the following, we will discuss the conceptual problems which prevented the formulation of such a bosonic DMFT and how they can be overcome.

\section{Lattice bosons in infinite dimensions: Different scaling for Bose-Einstein condensed and normal bosons}

A macroscopically large number of bosons can condense into a single quantum state. This BEC may be detected in the spectral decomposition of the one-particle density matrix,

$$
\rho_{i j}=\left\langle b_{i}^{\dagger} b_{j}\right\rangle=\sum_{\alpha} \lambda_{\alpha} \phi_{i \alpha}^{*} \phi_{j \alpha},
$$

where $b_{i}^{\dagger}$ and $b_{i}$ are creation and annihilation operators, respectively, for a boson at a lattice site $i$, with $\phi_{i \alpha}$ as the corresponding wave function. For simplicity, we discuss here only a single species of bosons so that the index $\nu$ can be omitted. When BEC occurs, one of the eigenvalues becomes macroscopically large, $\lambda_{0}=N_{0} \sim O(N)$, where $N$ is the total number of bosons. The density matrix then decomposes into

$$
\rho_{i j}=N_{0} \phi_{i 0}^{*} \phi_{j 0}+\tilde{\rho}_{i j},
$$

where the second term corresponds to the noncondensed, "normal" bosons. The first term has the remarkable property that it does not decrease even at large distance $R_{i j}=\| \mathbf{R}_{i}$ $-\mathbf{R}_{j} \|$ between the bosons at sites $i$ and $j$. Here, $\|\mathbf{R}\|$ denotes the length of $\mathbf{R}$ obtained by counting the minimal number of links between two sites on a lattice. By contrast, the second term in $\rho_{i j}$ decreases with increasing $R_{i j}$.

This has immediate consequences for the kinetic part of the Hamiltonian

$$
H_{0}=-t \sum_{\langle i j\rangle} b_{i}^{\dagger} b_{j}
$$

with $-t$ as the amplitude for hopping between nearest neighbor sites $i$ and $j$. For a uniform BEC with density $n_{0}$ $=N_{0} / N_{L}$, where $N_{L}$ is the number of lattice sites, one has $\phi_{i 0}^{*} \phi_{j 0}=1 / N_{L}$ such that the kinetic energy is given by 


$$
E_{\mathrm{kin}}=-t \sum_{\langle i j\rangle} \rho_{i j}=E_{\mathrm{kin}}^{\mathrm{BEC}}+E_{\mathrm{kin}}^{\mathrm{normal}},
$$

where

$$
E_{\mathrm{kin}}^{\mathrm{BEC}}=-t \sum_{\langle i j\rangle} N_{0}, E_{\mathrm{kin}}^{\mathrm{normal}}=-t \sum_{\langle i j\rangle} \tilde{\rho}_{i j}
$$

To derive a mean-field theory for lattice bosons via the limit of high spatial dimensions, the energy density $E_{\mathrm{kin}} / N_{L}$ needs to remain finite for $d$ or $Z \rightarrow \infty$. Since the energy density of the condensate, $E_{\mathrm{kin}}^{\mathrm{BEC}} / N_{L}=Z t n_{0}$, is proportional to $Z$, a nontrivial limit $Z \rightarrow \infty$ is obtained only if the hopping amplitude is scaled as $t=\widetilde{t} / Z$, with $\tilde{t}=$ const. ${ }^{17,18}$ In the case of normal lattice bosons (or fermions), the situation is characteristically different. Since $\widetilde{\rho}_{i j}$ is the transition amplitude for the hopping of a boson from $j$ to one of the $Z$ neighboring sites $i$, the respective normalized hopping probability is $\left|\tilde{\rho}_{i j}\right|^{2} \propto 1 / Z$, whence $\tilde{\rho}_{i j} \sim 1 / \sqrt{Z}$. For the energy density of the normal bosons, $E_{\mathrm{kin}}^{\text {normal }} / N_{L} \propto Z t \widetilde{\rho}_{i j}$, to remain finite for $Z \rightarrow \infty$, the hopping amplitude must therefore be scaled as in the case for fermions, namely, as $t=\tilde{t} / \bar{Z}^{26}$ In the more general case of hopping between sites $i$ and $j$ which are not nearest neighbors, the amplitudes $t_{i j}$ have to be scaled as

$$
t_{i j}=\widetilde{t}_{i j} /\left(Z^{R_{i j}}\right)^{s},
$$

where $s=1$ ("integer scaling") if the bosons are quantum condensed and $s=1 / 2$ ("fractional scaling") if they are in the normal state.

The total energy of a single species $(\nu=1)$ of correlated lattice bosons described by Hamiltonian (1) is given by

$$
E=-t \sum_{\langle i, j\rangle} N_{0}-t \sum_{\langle i, j\rangle} \widetilde{\rho}_{i j}+\frac{1}{2} U \sum_{i}\left\langle n_{i}\left(n_{i}-1\right)\right\rangle .
$$

If the scaling of the hopping amplitudes in the limit $Z \rightarrow \infty$ is performed on the level of the Hamiltonian (or the energy $E$ ), two cases have to be distinguished:

(i) $N_{0}=0$ : In the absence of a BEC, fractional scaling $(t$ $=\widetilde{t} / \sqrt{Z}$ ) has to be employed to arrive at a finite value of $E$ for $Z \rightarrow \infty$. We note that the interaction is purely local and hence independent of the spatial dimension of the system; consequently, $U$ need not be scaled at all.

(ii) $N_{0} \neq 0$ : In this case, integer scaling $(t=\tilde{t} / Z)$ has to be employed. Thereby, the contribution of the condensate [first term in Eq. (8)] remains. However, at the same time, the contribution of the noncondensed (normal) bosons to the kinetic energy is suppressed $\propto 1 / \sqrt{Z}$. The normal bosons thus become immobile. It can be shown rigorously that the meanfield equations obtained in this way are equivalent to those derived by Fisher et al., ${ }^{13}$ which are known to be exact in the limit of infinite-range hopping (see Sec. IV C).

The above discussion shows that the construction of a mean-field theory via the limit $Z \rightarrow \infty$, which is based on a scaling of the hopping amplitudes in the Hamiltonian, is either restricted to the normal state or removes the normal bosons from the problem. This is unsatisfactory since the important dynamical coupling of normal and condensed bosons is then eliminated from the outset. During the last 15

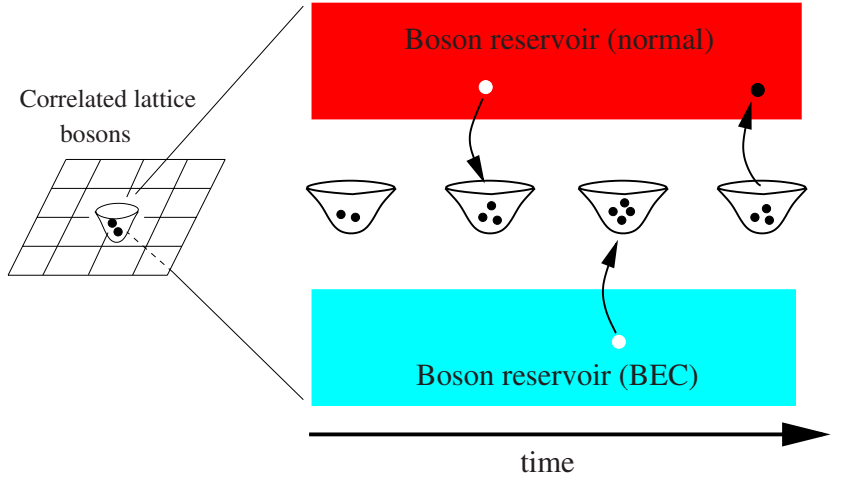

FIG. 1. (Color online) Bosonic dynamical mean-field theory (BDMFT): within the B-DMFT, the full many-body lattice problem is reduced to a single-site problem which is coupled to two reservoirs corresponding to bosons in the Bose-Einstein condensate and in the normal state. The integer occupation of the site changes in time and is determined by the local interactions and the time-dependent properties of the particle reservoirs. Although the total number of bosons is preserved, particles are scattered between the normal and the condensate reservoirs via the single site as shown by arrows. This schematic picture visualizes the idea of DMFT for lattice bosons in analogy to the fermionic counterpart described in Ref. 29.

years, the problem of how to scale the hopping amplitudes without eliminating the normal bosons presented an unsurmountable obstacle for the formulation of a bosonic DMFT. Indeed, our discussion shows that such a theory cannot be formulated on the level of a fully microscopic Hamilton operator, i.e., without making an additional Bogoliubov meanfield-type assumption. ${ }^{35}$

At this point, it should be pointed out that there exists no a priori condition according to which the scaling of the hopping amplitudes has to be performed in the Hamiltonian. Indeed, for the free energy of the model to remain finite in the limit $Z \rightarrow \infty$, the scaling can equally be performed in the partition function (or in the action entering in the functional integral which determines the partition function), from which the free energy is calculated.

\section{BOSONIC DYNAMICAL MEAN-FIELD THEORY}

\section{A. General structure of the bosonic dynamical mean-field theory}

We will now show that the long-standing problem of the scaling of the hopping amplitudes with the coordination number $Z$ or the dimension $d$ can be resolved by considering the large dimensional limit not in the Hamiltonian but in the action determining the Lagrangian density. Namely, integer scaling is employed whenever a hopping amplitude is associated with anomalous expectation values $\left\langle b_{i}\right\rangle$ and $\left\langle b_{i}^{\dagger}\right\rangle$ while fractional scaling is used otherwise. The B-DMFT obtained in this way treats normal and condensed bosons on equal footing and is thus able to describe both phases, including the transition between them, in a thermodynamically consistent way.

The general structure of the B-DMFT is shown in Fig. 1. In the limit $d \rightarrow \infty$, the bosonic many-body lattice problem is 
mapped onto a single-site problem with integer occupation. This site is coupled to two-particle reservoirs: one representing the normal and the other the quantum condensed bosons. These reservoirs represent the Weiss-type molecular fields of the B-DMFT. Their properties are determined selfconsistently by the B-DMFT equations. The particles hop onto and off the site as a function of time, thus changing the total number of bosons of the reservoirs. Therefore, local correlations lead to a dynamical depletion or filling of the condensate.

\section{B. Bosonic dynamical mean-field theory equations}

Here, we present the self-consistency equations of the B-DMFT for model (1); their derivation is discussed in detail in Appendix A. The time evolution of $\nu$ bosons on the single site $i=0$ is represented by the local propagator (Green's function)

$$
\mathbf{G}_{\nu}(\tau)=-\left\langle T_{\tau} \mathbf{b}_{\nu}(\tau) \mathbf{b}_{\nu}^{\dagger}(0)\right\rangle_{S_{\mathrm{loc}}},
$$

where we used the imaginary time, the finite temperature formalism, and the Nambu notation $\mathbf{b}_{\nu}^{\dagger} \equiv\left(b_{\nu}^{*}, b_{\nu}\right) ; T_{\tau}$ is the time ordering operator. The diagonal elements of the Green's function matrix in the Nambu space represent the quantummechanical probability amplitude for creating a boson on a site at one particular time and annihilating it after a time $\tau$ or the time inverted process. The off-diagonal elements, present only in the BEC phase, represent the amplitudes for creating or annihilating two bosons at different times. In the pathintegral formalism, the probabilities of such processes are determined by the local action, which in the B-DMFT is given by

$$
\begin{aligned}
S_{\mathrm{loc}}= & -\int_{0}^{\beta} d \tau \int_{0}^{\beta} d \tau^{\prime} \sum_{\nu} \mathbf{b}_{\nu}^{\dagger}(\tau) \mathcal{G}_{\nu}^{-1}\left(\tau-\tau^{\prime}\right) \mathbf{b}_{\nu}\left(\tau^{\prime}\right) \\
& +\int_{0}^{\beta} d \tau \sum_{\mu \nu} \frac{U_{\mu \nu}}{2} n_{\mu}(\tau)\left[n_{\nu}(\tau)-\delta_{\mu \nu}\right] \\
& +\int_{0}^{\beta} d \tau \sum_{\nu} \kappa_{\nu} \Phi_{\nu}^{\dagger}(\tau) \mathbf{b}_{\nu}(\tau) .
\end{aligned}
$$

Here, $\kappa_{\nu}$ is a numerical factor depending on the lattice structure, i.e., $\kappa_{\nu} \equiv \sum_{i \neq 0} \vec{t}_{i 0}^{\nu} / Z^{R_{i 0}}$ for $d \rightarrow \infty$ and $\kappa_{\nu} \equiv \sum_{i \neq 0} t_{i 0}^{\nu}$ for an approximation in finite dimensions. The free ("Weiss"28) mean-field propagator $\mathcal{G}_{\nu}^{-1}$, which is determined by the properties of the reservoir of normal $\nu$ bosons, is related to the local propagator $\mathbf{G}_{\nu}$ by the Dyson equation,

$$
\mathcal{G}_{\nu}^{-1}\left(i \omega_{n}\right)=\mathbf{G}_{\nu}^{-1}\left(i \omega_{n}\right)+\boldsymbol{\Sigma}_{\nu}\left(i \omega_{n}\right) \equiv\left(i \omega_{n} \sigma_{3}+\mu_{\nu} \mathbf{1}\right)-\boldsymbol{\Delta}_{\nu}\left(i \omega_{n}\right),
$$

where $\mathcal{G}$ and $\boldsymbol{\Sigma}$ are also matrices in the Nambu space. Here, $\omega_{n}=2 \pi n / \beta$ are even Matsubara frequencies with the inverse temperature $\beta=1 / k_{B} T$ and $\Sigma_{\mu}\left(i \omega_{n}\right)$ is the momentumindependent (local) self-energy. The quantity $\boldsymbol{\Delta}_{\nu}$ describes the resonant broadening of quantum-mechanical states on a lattice site and may be interpreted as a hybridization of bosons on that site with the surrounding bosonic bath. This hybridization function is determined by the local correlations through Eq. (10). The third term in Eq. (10) describes the coupling of a local boson to the condensate, the latter being represented by an order parameter $\boldsymbol{\Phi}_{\nu}^{\dagger}(\tau)$. In our formulation, this term arises naturally in the case of BEC and does not require a Bogoliubov substitution. ${ }^{35}$

The second B-DMFT equation is given by the lattice Hilbert transform,

$$
\mathbf{G}_{\nu}\left(i \omega_{n}\right)=\sum_{\mathbf{k}}\left\{\left[i \omega_{n} \sigma_{3}-\left(\epsilon_{\mathbf{k}}^{\nu}-\mu_{\nu}\right) \mathbf{1}\right]-\mathbf{\Sigma}_{\nu}\left(i \omega_{n}\right)\right\}^{-1},
$$

where $\epsilon_{\mathrm{k}}^{\nu}$ is the dispersion relation of noninteracting $\nu$ bosons, $\mu_{\nu}$ the chemical potential, $\mathbf{k}$ the wave vector, 1 a unity matrix, and $\sigma_{3}$ the Pauli matrix with \pm 1 on the diagonal. ${ }^{27,28}$ Equations (9) and (12) are the counterparts to the self-consistency equations of the DMFT for correlated lattice fermions. However, here these equations contain the condensate wave function $\boldsymbol{\Phi}_{\nu}$, i.e., the order parameter of the BEC, which enters as a source field in the action [Eq. (10)]. It can be determined exactly by calculating the average

$$
\boldsymbol{\Phi}_{\nu}(\tau)=\left\langle\mathbf{b}_{\nu}(\tau)\right\rangle_{S_{\text {loc }}}
$$

together with Eqs. (9) and (12). We note that in equilibrium, the time dependence enters via a trivial exponential factor $e^{-\mu_{\nu} \tau}$ which can be eliminated by a gauge transformation. ${ }^{36}$

Equations (9)-(13) constitute the B-DMFT solution of the generalized bosonic Hubbard model [Eq. (1)]. These equations are exact in the $d \rightarrow \infty$ limit and provide a comprehensive, thermodynamically consistent and conserving approximation in finite dimensions. In other words, the B-DMFT derived here is the first mean-field theory for correlated lattice bosons, which has all the attractive features characterizing the now well-established fermionic DMFT. ${ }^{28,29}$ In particular, the B-DMFT can be expected to be the best approximation to many-boson problems with strong local correlations since the on-site quantum fluctuations of the spin or density are treated exactly. Spatial correlations are neglected but can be restored, e.g., within cluster extensions of the B-DMFT. Furthermore, long-range ordered phases can be described within the B-DMFT by properly choosing the self-consistency conditions in analogy with the fermionic case. $^{28}$

\section{Bosonic dynamical mean-field theory and Gross-Pitaevskii equation}

The exact Euler-Lagrange equation of motion for the field $b(\tau)$ is obtained from the stationary conditions $\delta S_{\text {loc }}\left[b_{\nu}^{\dagger}, b_{\nu}\right] / \delta b_{\nu}^{\dagger}=0$ of the local B-DMFT action and is given by

$$
\begin{gathered}
\partial_{\tau} b_{\nu}(\tau)-\int_{0}^{\beta} d \tau^{\prime}\left[\Delta_{\nu}^{11}\left(\tau-\tau^{\prime}\right) b_{\nu}\left(\tau^{\prime}\right)+\Delta_{\nu}^{12}\left(\tau-\tau^{\prime}\right) b_{\nu}^{\dagger}\left(\tau^{\prime}\right)\right] \\
+\kappa_{\nu} b_{\nu}(\tau)+\sum_{\mu} U_{\mu \nu} b_{\mu}^{\dagger}(\tau) b_{\mu}(\tau) b_{\nu}(\tau)=\mu_{\nu} b_{\nu}(\tau) .
\end{gathered}
$$

If one replaces each field $b(\tau)$ by its expectation value (the order parameter $\Phi_{\nu}$ ), one arrives at 


$$
\begin{gathered}
\partial_{\tau} \Phi_{\nu}(\tau)-\int_{0}^{\beta} d \tau^{\prime}\left[\Delta_{\nu}^{11}\left(\tau-\tau^{\prime}\right) \Phi_{\nu}\left(\tau^{\prime}\right)+\Delta_{\nu}^{12}\left(\tau-\tau^{\prime}\right) \Phi_{\nu}^{*}\left(\tau^{\prime}\right)\right] \\
+\kappa_{\nu} \Phi_{\nu}(\tau)+\sum_{\mu} U_{\mu \nu}\left|\Phi_{\mu}(\tau)\right|^{2} \Phi_{\nu}(\tau)=\mu_{\nu} \Phi_{\nu}(\tau)
\end{gathered}
$$

This is a generalization of the standard Gross-Pitaevskii mean-field equation, a nonlinear differential equation for the condensed bosons which can be derived within the timedependent Hartree-Fock approximation. ${ }^{35}$ At present, it is not clear whether the replacement $b_{\nu}(\tau) \rightarrow \Phi_{\nu}(\tau)$, i.e., the factorization of the correlation function, holds rigorously as in other mean-field theories. ${ }^{37-40}$ Equation (15) is the classical equation of motion of the condensate of lattice bosons in the $d \rightarrow \infty$ limit. The second term on the left-hand side of Eq. (15) describes the retarding effects of normal bosons on the condensate and makes the generalized Gross-Pitaevskii equation a nonlinear integrodifferential equation. We note that in the standard Gross-Pitaevskii equation, the hybridization term $\boldsymbol{\Delta}_{\nu}$ is missing. Once $\boldsymbol{\Delta}_{\nu}$ has been determined by solving the B-DMFT self-consistency, Eqs. (15) can be used to determine $\Phi_{\nu}(\tau)$ and then calculate any response function of the condensate due to external perturbations, e.g., to describe the Bragg scattering. In equilibrium, $\Phi_{\nu}$ can be expected to be independent of $\tau$. Then, the stationary solution of Eq. (15) is easily obtained by solving a set of linear equations. For example, for the spinless bosonic Hubbard model (no index $\nu)$, we obtain $|\Phi|^{2}=\left[\mu-\kappa+\Delta^{11}\left(\omega_{n}=0\right)+\Delta^{12}\left(\omega_{n}=0\right)\right] / U$. The condensate density depends explicitly on the zero mode components of the hybridization functions for the normal subsystem.

\section{BOSONIC DYNAMICAL MEAN-FIELD THEORY IN DIFFERENT LIMITS OF PARAMETER SPACE}

The B-DMFT is a comprehensive mean-field theory for correlated bosons on a lattice, which means that the theory is valid for all input parameters and temperatures. This is an essential prerequisite for obtaining a reliable, approximate description of those parts of the phase diagram which cannot be studied perturbatively. Above all, the B-DMFT reproduces all known results obtained in special limits of the parameter space as depicted in Fig. 2. In particular, the exactly solvable limits of free and immobile bosons and of wellknown static mean-field approximations can be obtained directly from the B-DMFT. In the following, we discuss these limits in detail.

\section{A. Free bosons}

In the noninteracting limit, $U_{\mu \nu}=0$, the problem is trivially solvable in all dimensions. Since there is no interaction, all correlation functions factorize and the cavity method employed in Appendix A becomes exact. In this case, the BEC is described within the grand-canonical ensemble by a nonvanishing order parameter $\Phi$. However, the off-diagonal Green's (hybridization) functions of the normal bosons are zero. Explicitly, the local action has a bilinear (Gaussian) form,

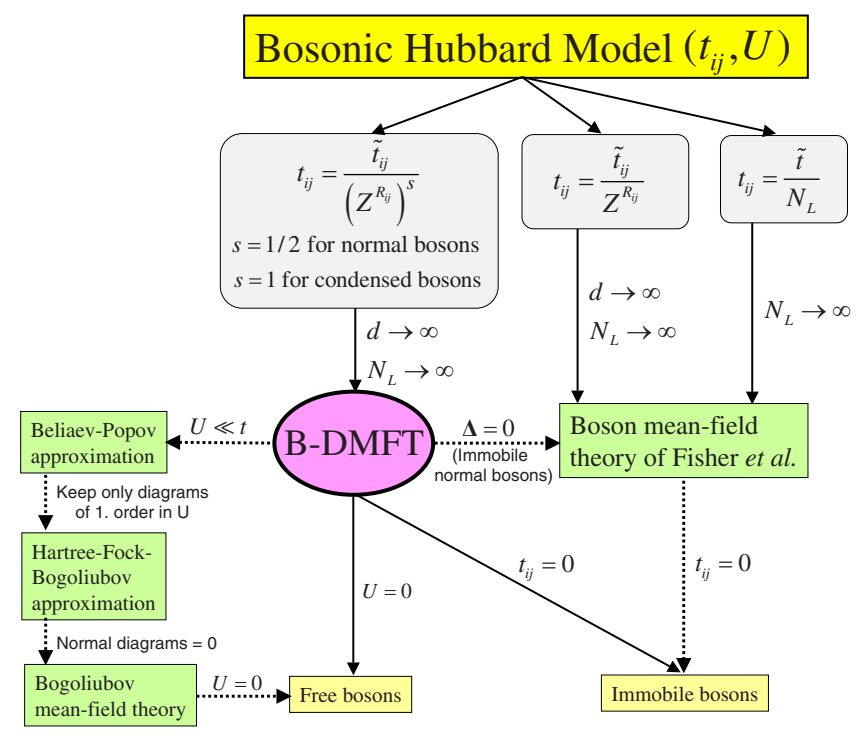

FIG. 2. (Color online) Relation of the B-DMFT to other approximations and exact limits; see Sec. IV.

$$
\begin{aligned}
S_{\text {loc }}^{\text {noninteracting }}= & -\int_{0}^{\beta} d \tau \int_{0}^{\beta} d \tau^{\prime} \sum_{\nu} \mathbf{b}_{\nu}^{\dagger}(\tau) \mathcal{G}_{\nu}^{-1}\left(\tau-\tau^{\prime}\right) \mathbf{b}_{\nu}\left(\tau^{\prime}\right) \\
& +\int_{0}^{\beta} d \tau \sum_{v} \kappa_{\nu} \Phi_{\nu}^{\dagger}(\tau) \mathbf{b}_{\nu}(\tau),
\end{aligned}
$$

with $\mathcal{G}_{\nu}^{-1}\left(\tau-\tau^{\prime}\right)=\left(-\partial_{\tau} \sigma_{3}+\mu_{\nu} \mathbf{1}\right) \delta\left(\tau-\tau^{\prime}\right)-\Delta_{\nu}\left(\tau-\tau^{\prime}\right)$. All the known equations, e.g., that for the particle number or the compressibility, can be easily derived from this action (see Appendix B). We note that in the noninteracting limit, the hopping amplitudes need not be scaled for $d<\infty$. However, to obtain a meaningful limit $d \rightarrow \infty$, the scaling scheme introduced in this paper is necessary. Otherwise, the condensate and normal bosons would not be treated on equal footing. That is, if only fractional scaling is employed, one obtains spurious infinities in the condensate phase, whereas if only integer scaling is used, the normal bosons (which contribute significantly at temperatures close or above $T_{\mathrm{BEC}}$ ) become immobile.

\section{B. Immobile bosons ("atomic limit")}

In the atomic limit, $t_{i j}^{\nu}=0$, all lattice sites are decoupled and the particles are immobile. In this case, the order parameter $\boldsymbol{\Phi}_{\nu}=0$, since no condensation is possible, and also the hybridization function $\boldsymbol{\Delta}_{\nu}=0$. For arbitrary dimensions, the exact action is then given by a sum over all equivalent sites with the same local action,

$$
\begin{aligned}
S_{\mathrm{loc}}^{\text {atomic }}= & -\int_{0}^{\beta} d \tau \sum_{\nu} \mathbf{b}_{\nu}^{\dagger}(\tau)\left(-\partial_{\tau} \sigma_{3}+\mu_{\nu} \mathbf{1}\right) \mathbf{b}_{\nu}(\tau) \\
& +\int_{0}^{\beta} d \tau \sum_{\mu \nu} \frac{U_{\mu \nu}}{2} n_{\mu}(\tau)\left[n_{\nu}(\tau)-\delta_{\mu \nu}\right],
\end{aligned}
$$

as obtained from the action [Eq. (10)] with $\boldsymbol{\Phi}_{\nu}=0$ and $\Delta_{\nu}(\tau)=0$ in $\mathcal{G}_{\nu}^{-1}\left(\tau-\tau^{\prime}\right)=\left(-\partial_{\tau} \sigma_{3}+\mu_{\nu} \mathbf{1}\right) \delta\left(\tau-\tau^{\prime}\right)-\Delta_{\nu}\left(\tau-\tau^{\prime}\right)$ 


\section{Mean-field theory of Fisher et al. (Ref. 13)}

The mean-field field theory of Fisher et al. ${ }^{13}$ is known to be the exact solution of the bosonic Hubbard model when the hopping amplitude is independent of distance and is scaled with the number of lattice sites $N_{L}$, i.e., $t_{i j}=\tilde{t} / N_{L} \cdot{ }^{13,41}$ This is also called the limit of infinite-range hopping. ${ }^{13,41,42}$ The free energy density of the bosonic Hubbard model in this limit has the form

$$
\begin{aligned}
F^{\text {infinite-range }}= & F_{a t}-k T \ln \left\langleT _ { \tau } \left[\operatorname { e x p } \left(-\int_{0}^{\beta} d \tau \sum_{\nu} \kappa_{\nu}\right.\right.\right. \\
& \left.\left.\left.\times \boldsymbol{\Phi}_{\nu}^{\dagger} \mathbf{b}_{\nu}(\tau)\right)\right]\right\rangle_{H_{\text {at }}}-\sum_{\nu} \kappa_{\nu}\left|\Phi_{\nu}\right|^{2} .
\end{aligned}
$$

Here, the atomic part $F_{\text {at }}=-k T \ln \operatorname{Tr} \exp \left(-\beta H_{\mathrm{at}}\right)$ is given by the Hamiltonian $H_{\mathrm{at}}$, which is obtained from the lattice Hamiltonian by setting all hopping amplitudes equal to zero. The average in the second term is taken with respect to $H_{\mathrm{at}}$. The stationarity condition for the free energy [Eq. (18)] with respect to $\Phi_{\nu}$, i.e., $\partial F^{\text {infinite-range } /} \partial \boldsymbol{\Phi}_{\nu}=0$, then yields the selfconsistent mean-field equation,

$$
\kappa_{\nu} \boldsymbol{\Phi}_{\nu}=k T \frac{\left\langle T_{\tau} \mathbf{b}_{\nu}\left[\exp \left(-\int_{0}^{\beta} d \tau \sum_{\nu} \kappa_{\nu} \boldsymbol{\Phi}_{\nu}^{\dagger} \mathbf{b}_{\nu}(\tau)\right)\right]\right\rangle_{H_{\mathrm{at}}}}{\left\langle T_{\tau}\left[\exp \left(-\int_{0}^{\beta} d \tau \sum_{\nu} \kappa_{\nu} \boldsymbol{\Phi}_{\nu}^{\dagger} \mathbf{b}_{\nu}(\tau)\right)\right]\right\rangle_{H_{\mathrm{at}}}} .
$$

Since the normal bosons are immobile, and thus not dynamically coupled to the condensed bosons, the theory of Fisher et al. ${ }^{13}$ is a static mean-field theory.

We now show that the mean-field [Eq. (19)] can also be obtained in the $d \rightarrow \infty$ limit as put forward in Refs. 15, 17, and 18. Indeed, by employing the cavity method for lattice bosons and using only integer scaling for the hopping amplitudes, the local action in the $d \rightarrow \infty(Z \rightarrow \infty)$ limit takes the form

$$
\begin{aligned}
S_{\mathrm{loc}}^{d \rightarrow \infty, \text { integer scaling }}= & -\int_{0}^{\beta} d \tau \sum_{\nu} \mathbf{b}_{\nu}^{\dagger}(\tau)\left(-\partial_{\tau} \sigma_{3}+\mu_{\nu} \mathbf{1}\right) \mathbf{b}_{\nu}(\tau) \\
& +\int_{0}^{\beta} d \tau \sum_{\mu \nu} \frac{U_{\mu \nu}}{2} n_{\mu}(\tau)\left[n_{\nu}(\tau)-\delta_{\mu \nu}\right] \\
& +\int_{0}^{\beta} d \tau \sum_{\nu} \kappa_{\nu} \boldsymbol{\Phi}_{\nu}^{\dagger}(\tau) \mathbf{b}_{\nu}(\tau)
\end{aligned}
$$

This expression differs from the local action in the atomic limit [Eq. (17)] by the presence of the last term which describes the condensate. However, in equilibrium, the BEC order parameter is time independent in which case Eq. (20) yields the free energy density as

$$
\begin{aligned}
F^{d \rightarrow \infty, \text { integer scaling }}= & F_{\text {at }}-k T \ln \left\langleT _ { \tau } \operatorname { e x p } \left(-\int_{0}^{\beta} d \tau\right.\right. \\
& \left.\left.\times \sum_{\nu} \kappa_{\nu} \boldsymbol{\Phi}_{\nu}^{\dagger} \mathbf{b}_{\nu}(\tau)\right)\right\rangle_{H_{\text {at }}} .
\end{aligned}
$$

The BEC order parameter $\boldsymbol{\Phi}_{\nu}$ obeys the self-consistent equation,

$$
\boldsymbol{\Phi}_{\nu}=\frac{1}{Z_{\text {loc }}^{d \rightarrow \infty, \text { integer scaling }}} \cdot \int D\left[b, b^{*}\right] \mathbf{b}_{\nu} e^{-S_{\text {loc }}^{d \rightarrow \infty, \text { integer scaling }}\left[b, b^{*} ; \boldsymbol{\Phi}_{\nu}\right]} .
$$

Other correlation functions and observables can be determined similarly.

The free energy density [Eq. (21)] is seen to differ from Eq. (18) only by the absence of the last term proportional to the density of the condensate. Nevertheless, the equations for the BEC order parameter as well as correlation functions and observables are the same. Thus, the approximation of constant hopping amplitude (infinite-range hopping) and the $d$ $\rightarrow \infty$ limit with integer scaling give rise to the same meanfield equations. At $T=0$, these equations can also be derived by yet another approximation, namely, a variational method using a Gutzwiller-type wave function. ${ }^{15,43}$ The mean-field theory of Fisher et al. ${ }^{13}$ and its generalization to spinful bosons were widely used to investigate quantum phase transitions and the phase diagrams of correlated lattice boson systems and of mixtures of lattice bosons and fermions. ${ }^{3-8,44-46}$

Equation (21) and (22) can also be obtained directly from the B-DMFT self-consistency equations by neglecting the hybridization function, i.e., by setting $\boldsymbol{\Delta}_{\nu}=0$. Then, the local action of the B-DMFT [Eq. (10)] is the same as that in Eq. (20). Furthermore, Eq. (12) is satisfied automatically in this limit since only the state with $\mathbf{k}=0$ is taken into account. It should be noted, however, that in the absence of the hybridization function $\boldsymbol{\Delta}_{\nu}$, the noninteracting limit of the normal bosons cannot be reproduced; i.e., the mean-field theory of Fisher et $a l .^{13}$ does not describe the limit of free, normal bosons.

\section{Weak-coupling (Bogoliubov) mean-field theory}

A perturbation expansion to first order in $U_{\mu \nu}$ is equivalent to the Hartree-Fock-Bogoliubov approximation with the static self-energy $\Sigma_{\nu}^{11}=2 \Sigma_{\mu} U_{\nu \nu} \bar{n}_{\mu}^{\mathrm{BEC}}-2 \Sigma_{\omega_{n}, \mu} U_{\nu \mu} \mathcal{G}_{\mu}\left(\omega_{n}\right) / \beta$ and $\Sigma_{\nu}^{12}=\Sigma_{\mu} U_{\nu \mu} \bar{n}_{\mu}^{\mathrm{BEC}}$. For such a self-energy, the selfconsistency condition [Eq. (12)] is equivalent to the selfconsistent Hartree-Fock-Bogoliubov approximation (sometimes called the "first-order Popov" approximation). ${ }^{35,36}$ This self-consistent approximation is known to lead to a gapped spectrum in the condensed phase because off-diagonal elements in the self-energy are calculated in higher order due to self-consistency. By contrast, the Bogoliubov approximation $^{35,36}$ is obtained if only particular diagrams corresponding to the self-energies $\Sigma_{\nu}^{11}=2 \Sigma_{\mu} U_{\nu \mu} \bar{n}_{\mu}^{\mathrm{BEC}}$ and $\Sigma_{\nu}^{12}=\Sigma_{\mu} U_{\nu \mu} \bar{n}_{\mu}^{\mathrm{BEC}}$ are taken into account.

The second-order expansion contains many diagrams (see Refs. 35 and 36). Checking term by term, we find that the 
B-DMFT reproduces the Beliaev-Popov approximation (sometimes called the "second-order Popov approximation" ${ }^{35}$ if, in addition, in the latter approximation only local irreducible self-energy diagrams (consistent with the $d \rightarrow \infty$ limit) are retained.

\section{BOSONIC DYNAMICAL MEAN-FIELD THEORY SOLUTION OF THE BOSONIC FALICOV-KIMBALL MODEL}

We now apply the B-DMFT to study BEC in a mixture of two different species of bosons: itinerant $b$ bosons and immobile $f$ bosons. We assume the $b$ bosons not to interact with each other but only with $f$ bosons, while $f$ bosons interact also mutually, i.e., $U_{b b}=0, U_{b f}>0$, and $U_{f f}>0$ in Eq. (1). We call this the bosonic-Falicov-Kimball (BFK) model since it is a bosonic generalization of the Falicov-Kimball model for fermions, which has been widely studied in condensedmatter physics. ${ }^{47}$ Experimentally, such a system can be realized by loading an optical lattice either with a mixture of two different species of bosonic alkali atoms (e.g., ${ }^{7} \mathrm{Li}$ and ${ }^{87} \mathrm{Rb}$ ) or by one kind of atom with two different hyperspin states (i.e., with $F=1,2$ and specific values of the $z$ component of $F)$. In addition, the electric fields generating the potentials of the optical lattice and the external magnetic field controlling the Feshbach resonances should be tuned such that one species of particles is immobile and the other is noninteracting (or only weakly interacting). The realization of a fermionic Falicov-Kimball model by cold fermionic atoms in an optical lattice was discussed in Refs. 48 and 49.

It is important to note that, in spite of the immobility of the $f$ bosons, the BFK model is still a many-body problem because the immobile particles are thermodynamically coupled to the mobile particles by the interaction. In particular, the optimal configuration of the localized bosons depends on the interaction, temperature, and density of the particles. In the fermionic counterpart, one finds that the position of the immobile particles is either random or long-range ordered; phase separation between these two components can also occur. The numerical solution of the Falicov-Kimball model is limited to small lattices and requires an annealed average over a large number of configurations of immobile particles.

For the BFK model, the local impurity problem can be integrated analytically. The self-consistency equations can be then solved by standard numerical techniques. Since the $f$ bosons are immobile, their number on each site is conserved. Hence, the $f$-boson subsystem cannot undergo BEC and the occupation number operator $n_{f}$ of the single site becomes a classical variable with $n_{f}=0,1,2, \ldots$. The local action [Eq. (10)] is then quadratic in the bosonic operators. Consequently, the local propagator $G_{b}\left(i \omega_{n}\right)$ for $b$ bosons and the local partition function $Z_{\mathrm{loc}}\left(\mu_{b}, \mu_{f}\right)$, and thereby the BEC transition temperature $T_{\mathrm{BEC}}$ for the $b$ bosons, can be evaluated directly. The local partition function of the BFK model is determined by

$$
Z_{\mathrm{loc}}\left(\mu_{b}, \mu_{f}\right)=\sum_{n_{f}=0,1,2, \ldots}^{\infty} e^{\beta n_{f}\left(\mu_{f}-U_{f f} n_{f} / 2\right)} Z_{\mathrm{loc}}^{0}\left(\mu_{b}-U_{b f} n_{f}, \mu_{f}\right),
$$

where

$$
Z_{\mathrm{loc}}^{0}\left(\mu_{b}, \mu_{f}\right) \sim e^{-\kappa_{b}\left|\phi_{b}\right|^{2} / \mu_{b}-\Delta_{b}(0)} \prod_{\omega_{n}}\left(\frac{1}{i \omega_{n}+\mu_{b}-\Delta_{b}\left(i \omega_{n}\right)}\right)
$$

is the partition function for $U_{b f}=0$. The local propagator for normal $b$ bosons is given by

$$
G_{b}\left(i \omega_{n}\right)=\sum_{n_{f}=0,1,2, \ldots}^{\infty} \frac{w_{n_{f}}}{i \omega_{n}+\mu_{b}-U_{b f} n_{f}-\Delta_{b}\left(i \omega_{n}\right)} .
$$

Here,

$$
w_{n_{f}}=e^{\beta n_{f}\left(\mu_{f}-U_{f f} n_{f} / 2\right)} \frac{Z_{\mathrm{loc}}^{0}\left(\mu_{b}-U_{b f} n_{f}, \mu_{f}\right)}{Z_{\mathrm{loc}}\left(\mu_{b}, \mu_{f}\right)}
$$

is the probability for the single site to be occupied by exactly $n_{f}=0,1,2, \ldots$ bosons. For hard-core $f$ bosons $\left(U_{f f}=\infty\right)$, this leads to $w_{n_{f}=1}=\bar{n}_{f}$ and $w_{n_{f}=0}=1-\bar{n}_{f}$. In this case, $\bar{n}_{f}$ rather than $\mu_{f}$ is used as an independent thermodynamical variable. Propagator (25) describes quantum and thermal fluctuations of normal bosons. In the absence of the interaction between $b$ bosons, off-diagonal terms in the local propagator are zero. The Gross-Pitaevskii equation is then obviously exact and reduces to a homogeneous, linear equation of the form $\left[-i \omega_{n}-\mu_{b}+\kappa_{b}+\Delta_{b}\left(i \omega_{n}\right)\right] \phi_{b}\left(i \omega_{n}\right)=0$ for each Fourier component. For $\omega_{n} \neq 0$, the only solution is $\phi_{b}\left(\omega_{n} \neq 0\right)=0$. The static $\left(\omega_{n}=0\right)$ component of the BEC order parameter is finite if $\mu_{b}=\kappa_{b}+\Delta_{b}(0)$ and must be determined by fixing the average density of $b$ bosons.

A striking result obtained for this model is an enhancement of $T_{\mathrm{BEC}}$ for increasing repulsion between the $b$ and $f$ bosons, with a maximum of $T_{\mathrm{BEC}}$ at intermediate values of $U_{b f}$ and a saturation at large $U_{b f}$. This behavior is explicitly seen in Fig. 3(a) for hard-core $f$ bosons $\left(U_{f f}=\infty\right.$, i.e., $n_{f}$ $=0,1)$ on a simple cubic lattice where we plotted the relative change in $T_{\mathrm{BEC}}$ with respect to $T_{\mathrm{BEC}}^{0}$ in the noninteracting system. The increase in $T_{\mathrm{BEC}}$ is due to the blocking of a fraction of sites by heavy atoms, which increases the density of the $b$ bosons. However, this argument cannot explain the nonmonotonicity of $T_{\mathrm{BEC}}$ vs $U_{b f}$ shown in Fig. 3(a). In fact, the maximum is due to the correlation induced band splitting and the narrowing of the lower subband [see Fig. 3(c)] which lead to an increase and decrease in $T_{\mathrm{BEC}}$, respectively. Furthermore, at fixed temperature $T$, the average condensate density $\bar{n}_{b}^{\mathrm{BEC}}(T)$ is also found to increase with increasing repulsion [see Fig. 3(b)], although the interaction induced scattering between bosons usually removes particles from the condensate, thereby reducing its density. ${ }^{50}$ At zero temperature, all $b$ bosons are in the condensate. Similar results are found for other lattices (cf. Fig. 4).

These results originate from local correlations which are captured exactly by the B-DMFT but not by conventional approximations. The consequences can be inferred by considering the total density of $b$ bosons,

$$
\bar{n}_{b}=\bar{n}_{b}^{\mathrm{BEC}}(T)+\int d \omega \frac{A_{b}\left(\omega+\mu_{b}\right)}{\exp (\omega / T)-1} .
$$

The second term gives the contribution of normal $b$ bosons for which the spectral function $A_{b}(\omega)=-\operatorname{Im} G_{b}(\omega) / \pi$ is 

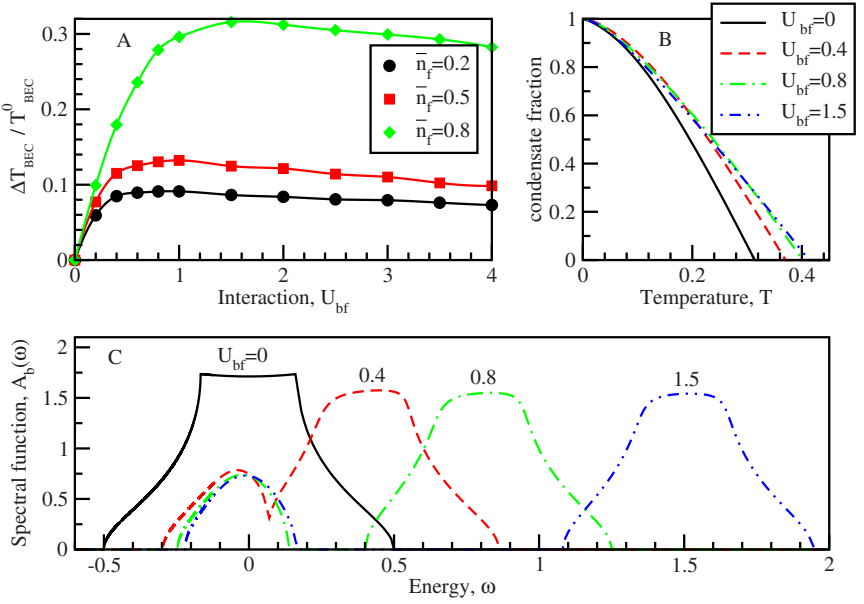

FIG. 3. (Color online) Bose-Einstein condensation of a mixture of itinerant and localized, correlated lattice bosons: (a) Enhancement of the BEC transition temperature with increasing interaction strength $U_{b f}$ in a two component boson mixture with different densities $\bar{n}_{f}$ of the localized $f$ bosons. (b) Dependence of the condensate fraction $\bar{n}_{b}^{\mathrm{BEC}}(T) / \bar{n}_{b}$ on temperature for different interactions $U_{b f}$ at $\bar{n}_{f}=0.8$. (c) Spectral functions for different values of $U_{b f}$ at $\bar{n}_{f}$ $=0.8$. The increase in $T_{\mathrm{BEC}}$ and the condensate fraction with increasing $U_{b f}$ and $\bar{n}_{f}$ is caused by correlation effects leading to a redistribution of the spectral weight for the $b$-bosonic subsystem. The correlation gap opens when $U_{b f}$ exceeds a critical value which depends on $\bar{n}_{f}$. The opening of the gap is not associated with a phase transition of the mobile bosons. Results are obtained for a three-dimensional cubic lattice with unit bandwidth and $\bar{n}_{b}=0.65$. In the hard-core limit, the spectral functions are temperature independent because the occupation probability of $f$ bosons is either $\bar{n}_{f}$ or $1-\bar{n}_{f}$.

shown in Fig. 3(c) for different $U_{b f}$ values. The spectral weight is seen to be strongly redistributed, forming lowerand upper-Hubbard subbands at low and high energies $\omega$, respectively, which are separated by the energy $U_{b f}$. We note that the splitting and rounding of the shapes are genuine correlation effects. The occupation probability of normal $b$ bosons, i.e., the Bose-Einstein distribution function, decays exponentially with increasing $\omega$. This implies an extremely small particle number in the upper-Hubbard subband at large $U_{b f}$. Since the total number of $b$ bosons is constant, the particles are necessarily transferred into the condensate. Hence, at fixed temperature, $\bar{n}_{b}^{\mathrm{BEC}}$ increases, thereby enhancing $T_{\mathrm{BEC}}$. The spectral weight contributing to the upper-Hubbard subband is also proportional to $\bar{n}_{f}$, implying that $T_{\mathrm{BEC}}$ increases with $\bar{n}_{f}$ too.

The B-DMFT prediction of an increase in $T_{\mathrm{BEC}}$ and in the condensate density due to local correlations are expected to be observable in mixtures of mobile and localized bosons on three-dimensional optical lattices. Such correlations can thus be employed in the laboratory to enhance $T_{\mathrm{BEC}}$ of bosonic condensates. We also note that on bipartite lattices with special densities of bosons, e.g., $\bar{n}_{f}=\bar{n}_{b}=0.5$, long-range order in the density of the $f$ subsystem and, in turn, a supersolid phase in the $b$ subsystem is expected to form. In such a phase periodically arranged, localized bosons will coexist with a condensate of mobile bosons whose density is likewise periodically modulated. Obviously, the physics of this seemingly simple bosonic model is extraordinarily rich.

\section{CONCLUSIONS}

In this paper, we derived the first comprehensive, thermodynamically consistent theoretical framework for the investigation of correlated lattice bosons - a B-DMFT. In analogy to its fermionic counterpart, the B-DMFT becomes exact in the limit of high spatial dimensions $d$ or coordination number $Z$ and may be employed to compute the phase diagram and thermodynamics of interacting lattice boson systems in the entire range of microscopic parameters. The B-DMFT requires a different scaling of the hopping amplitude with $Z$ depending on whether the system is in the normal or in the Bose-condensed phase. This additional difficulty compared to the fermionic case prevented the formulation of the B-DMFT in the past. As shown here, it can be overcome by performing the scaling not in the Hamiltonian but in the action. The B-DMFT equations consist not only of a bosonic single-impurity problem ${ }^{51}$ in the presence of a self-
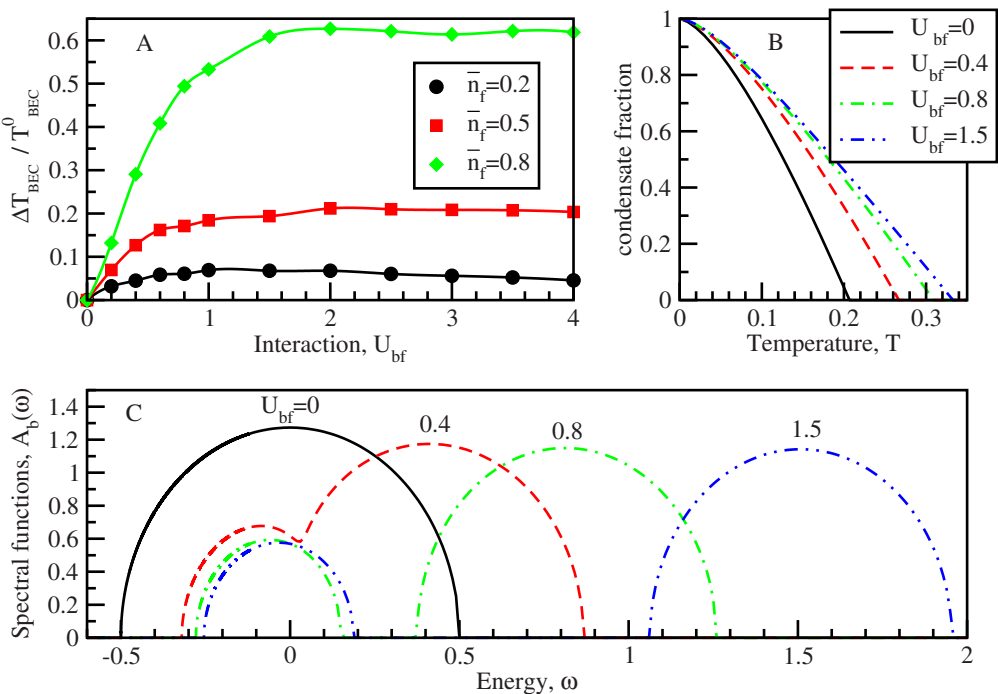

FIG. 4. (Color online) Bose-Einstein condensation of a mixture of itinerant and localized, correlated lattice bosons: In contrast to Fig. 2, results here are obtained for a Bethe lattice with infinite $Z$, unit bandwidth, and $\bar{n}_{b}=0.5$. They are exact for the bosonic-Falicov-Kimball model. (a) Enhancement of the BEC transition temperature with increasing interaction strength $U_{b f}$ in a two component boson mixture with different densities $\bar{n}_{f}$ of the localized $f$ bosons. (b) Dependence of the condensate fraction $\bar{n}_{b}^{\mathrm{BEC}}(T) / n_{b}$ on temperature for different interactions $U_{b f}$ at $\bar{n}_{f}=0.8$. (c) Spectral functions for different values of $U_{b f}$ at $\bar{n}_{f}$ $=0.8$. The increase in $T_{\mathrm{BEC}}$ and the condensate fraction with increasing $U_{b f}$ and $\bar{n}_{f}$ is caused by correlation effects leading to a redistribution of the spectral weight for the $b$-bosonic subsystem. 
consistency condition (the momentum integrated Dyson equation) but involve an additional coupling to the condensate wave function.

We documented the comprehensive nature of the B-DMFT by explicitly reproducing results previously obtained in special limits of parameter space and by deriving other bosonic mean-field theories. For example, by calculating the local self-energy of the B-DMFT in the weakcoupling regime, $U / t \ll 1$, in perturbation theory and by including all normal and anomalous terms to first order in $U / t$, one obtains the Hartree-Fock-Bogoliubov self-consistent mean-field approximation. ${ }^{35}$ By neglecting the anomalous terms, the standard Bogoliubov theory for lattice bosons is recovered. The inclusion of the second-order corrections to the local self-energy corresponds to the Beliaev-Popov approximation (with the additional assumption of a local selfenergy). Furthermore, by neglecting all terms containing the hybridization function in the local action, one obtains the mean-field theory developed in Refs. 13, 17, and 18, which corresponds to the exact solution of the bosonic Hubbard model [Eq. (1)] in the large dimension limit if only integer scaling is applied.

In contrast to the previous mean-field theories, the B-DMFT constructed here treats normal and condensed bosons on equal footing. In particular, the B-DMFT takes into account the effects due to finite hopping and dynamical broadening of the quantum levels. The inclusion of correlated normal bosons and their dynamic coupling to the condensate revises the results of static mean-field theories in several ways; in particular, these effects are as follows:

(i) modify the phase diagram of the spinless bosonic Hubbard model at finite temperatures (for example, the cusplike shape of the phase boundary between the Mott and the superfluid phases ${ }^{13,16,17}$ is expected to be changed or perhaps even eliminated by the appearance of an intermediate normal fluid and/or metallic phase);

(ii) change the condensation temperature $T_{\mathrm{BEC}}$;

(iii) renormalize two-particle correlation functions, which determine the compressibility and other susceptibilities, e.g., the magnetic susceptibility in the case of spinful bosons.

Furthermore, since the B-DMFT is applicable even in the noncondensed phase $\left(T>T_{\mathrm{BEC}}\right)$, it also provides a computational framework for investigations of normal bosons, e.g., of the Mott transition. Here, it is interesting to note that the correlation-induced broadening of the Hubbard $\delta$ peaks in the spectral functions implies that the Mott transition occurs already when these bands begin to overlap. All these issues are highly relevant for a comprehensive explanation of the on-going experiments with cold bosonic atoms in optical lattices ${ }^{4}$ but are not systematically address within the existing static mean-field theories.

We note that the B-DMFT is not merely a perturbative improvement of the static mean-field theory ${ }^{13,17,18,20}$ with respect to the hybridization since in the B-DMFT the hybridization function is included to all orders. We applied the B-DMFT to solve the bosonic-Falicov-Kimball model, i.e., a lattice model of itinerant and localized, interacting bosons. Due to the localized nature of the interacting bosons (providing a type of annealed disorder to the system since the localized particles are thermodynamically coupled to the itinerant bosons), the problem reduces to a set of algebraic equations. We find that local correlations enhance the transition temperature into the condensate and can thus be employed in the laboratory to increase $T_{\mathrm{BEC}}$.

In general, the effective single-site problem of a bosonic impurity coupled to two baths (the condensate and normal bosons) has to be solved numerically. The development of a reliable bosonic single-impurity solver ${ }^{52}$ is a challenging task, which took several years in the case of the fermionic DMFT. This process can also involve a formulation of the proper bosonic impurity Hamiltonian corresponding to the B-DMFT action derived here. One of the main goals of this paper is to present the foundations of a comprehensive meanfield theory for correlated bosons and thereby instigate further research by analytical and numerical means.

\section{ACKNOWLEDGMENTS}

We thank R. Bulla, W. Hofstetter, and M. Kollar for useful discussions. One of us (D.V.) is grateful to V. Janis for illuminating discussions during 1991-1992 on the scaling of the hopping amplitude of lattice bosons. This work was supported in part by the Sonderforschungsbereich 484 of the Deutsche Forschungsgemeinschaft (DFG).

\section{APPENDIX A: DERIVATION OF THE BOSONIC DYNAMICAL MEAN-FIELD THEORY SELF-CONSISTENCY EQUATIONS}

Here, we derive the B-DMFT equations for the generalized bosonic Hubbard Hamiltonian [Eq. (1)] by applying the cavity method. ${ }^{28}$ To this end, the partition function

$$
Z=\int D\left[b_{\nu}^{*}, b_{\nu}\right] \exp \left(-S\left[b_{\nu}^{*}, b_{\nu}\right]\right)
$$

is calculated within the grand-canonical ensemble, making use of the path-integral approach over complex coherent states. ${ }^{53}$ The action

$$
S\left[b_{\nu}^{*}, b_{\nu}\right]=\int_{0}^{\beta} d \tau\left[\sum_{i \nu} b_{i \nu}^{*}(\tau)\left(\partial_{\tau}-\mu\right) b_{i \nu}(\tau)+H(\tau)\right]
$$

is split into a single-site term with $i=0$,

$$
\begin{aligned}
S_{0}= & \int_{0}^{\beta} d \tau\left[\sum_{\nu} b_{0 \nu}^{*}(\tau)\left(\partial_{\tau}-\mu\right) b_{0 \nu}(\tau)\right. \\
& \left.+\frac{1}{2} \sum_{\mu \nu} U_{\mu \nu} n_{0 \mu}\left(n_{0 \nu}-\delta_{\mu \nu}\right)\right],
\end{aligned}
$$

a term representing the coupling between this site and the rest of the lattice $(i \neq 0)$, 


$$
\Delta S=\int_{0}^{\beta} d \tau \sum_{i \nu}\left(t_{0 i}^{\nu} b_{0 \nu}^{\dagger} b_{i \nu}+t_{i 0}^{\nu} b_{i \nu}^{\dagger} b_{0 \nu}\right) \equiv \int_{0}^{\beta} d \tau \Delta S(\tau),
$$

and a remaining part with site indices $i, j \neq 0$,

$$
S^{(0)}=\int_{0}^{\beta} d \tau\left[\sum_{i \neq 0 \nu} b_{i \nu}^{*}(\tau)\left(\partial_{\tau}-\mu\right) b_{i \nu}(\tau)+H^{(0)}(\tau)\right]
$$

such that

$$
S\left[b_{\nu}^{*}, b_{\nu}\right]=S_{0}+\Delta S+S^{(0)} .
$$

In the next step, we expand the exponential function with respect to the action $\Delta S$ and perform the functional integral over all variables with site indices $i \neq 0$. As a result, we obtain a formally infinite series with all possible manyparticle correlation functions, i.e.,

$$
\begin{aligned}
Z=\int D\left[b_{0 \nu}^{*}, b_{0 \nu}\right] e^{-S_{0}\left[b_{0 \nu}^{*}, b_{0 \nu}\right]} Z^{(0)}\left(1-\int_{0}^{\beta} d \tau\langle\Delta S(\tau)\rangle_{S^{(0)}}\right. \\
\left.+\frac{1}{2 !} \int_{0}^{\beta} d \tau_{1} \int_{0}^{\beta} d \tau_{2}\left\langle\Delta S\left(\tau_{1}\right) \Delta S\left(\tau_{2}\right)\right\rangle_{S^{(0)}}+\cdots\right),
\end{aligned}
$$

where $\langle\ldots\rangle_{S^{(0)}}$ denotes the average taken with respect to $S^{(0)}$ (the action where the site $i=0$ is excluded) and $Z^{(0)}$ is the corresponding partition function. In contrast to the fermionic case, ${ }^{28}$ there remain anomalous correlation functions in the Bose-condensed phase such as $\left\langle b_{i \nu}(\tau)\right\rangle_{S^{(0)}},\left\langle b_{i \nu}(\tau) b_{j \nu}\left(\tau^{\prime}\right)\right\rangle_{S^{(0)}}$, $\left\langle b_{i \nu}^{*}(\tau) b_{j \nu}\left(\tau^{\prime}\right) b_{k \nu}\left(\tau^{\prime \prime}\right)\right\rangle_{S^{(0)}}$, etc. The lowest first-order terms take the form

$$
\begin{aligned}
\int_{0}^{\beta} d \tau\langle\Delta S(\tau)\rangle_{S^{(0)}}= & \int_{0}^{\beta} d \tau \sum_{\nu} \sum_{j}^{\prime}\left[t_{0 j}^{\nu} b_{0 \nu}^{*}(\tau)\left\langle b_{\nu j}(\tau)\right\rangle_{S^{(0)}}\right. \\
& \left.+t_{j 0}^{\nu} b_{0 \nu}(\tau)\left\langle b_{j \nu}^{*}(\tau)\right\rangle_{S^{(0)}}\right]
\end{aligned}
$$

where the prime on the summation symbol indicates that the lattice indices are different from 0 , i.e., $j \neq 0$ in Eq. (A8). The second-order terms read

$$
\begin{aligned}
\frac{1}{2 !} \int_{0}^{\beta} d \tau_{1} \int_{0}^{\beta} d \tau_{2}\left\langle\Delta S\left(\tau_{1}\right) \Delta S\left(\tau_{2}\right)\right\rangle_{S^{(0)}=} & \frac{1}{2 !} \int_{0}^{\beta} d \tau_{1} \int_{0}^{\beta} d \tau_{2} \sum_{\nu} \sum_{j k}^{\prime}\left[t_{j 0}^{\nu} t_{k 0}^{\nu}\left\langle b_{j \nu}^{*}\left(\tau_{1}\right) b_{k \nu}^{*}\left(\tau_{2}\right)\right\rangle_{S(0)} b_{0 \nu}\left(\tau_{1}\right) b_{0 \nu}\left(\tau_{2}\right)\right. \\
& +t_{j 0}^{\nu} t_{0 k}^{\nu}\left\langle b_{j \nu}^{*}\left(\tau_{1}\right) b_{k \nu} \tau_{2}\right\rangle_{S^{(0)}} b_{0 \nu}\left(\tau_{1}\right) b_{0 \nu}^{*}\left(\tau_{2}\right)+t_{0 j}^{\nu} t_{k 0}^{\nu}\left\langle b_{j \nu}\left(\tau_{1}\right) b_{k \nu}^{*}\left(\tau_{2}\right)\right\rangle_{S^{(0)}} b_{0 \nu}^{*}\left(\tau_{1}\right) b_{0 \nu}\left(\tau_{2}\right) \\
& \left.+t_{0 j}^{\nu} t_{0 k}\left\langle b_{j \nu}\left(\tau_{1}\right) b_{k \nu}\left(\tau_{2}\right)\right\rangle_{S(0)} b_{0 \nu}^{*}\left(\tau_{1}\right) b_{0 \nu}^{*}\left(\tau_{2}\right)\right] .
\end{aligned}
$$

Higher-order terms are obtained similarly. Defining the (connected) correlation functions for the condensate,

$$
\begin{aligned}
& \phi_{j \nu}(\tau)=\left\langle b_{j \nu}(\tau)\right\rangle_{S^{(0)}}, \\
& \phi_{j \nu}^{*}(\tau)=\left\langle b_{j \nu}^{*}(\tau)\right\rangle_{S^{(0)}},
\end{aligned}
$$

Eq. (A8) can be written as

$$
\begin{aligned}
\int_{0}^{\beta} d \tau\langle\Delta S(\tau)\rangle_{S^{(0)}}= & \int_{0}^{\beta} d \tau \sum_{\nu} \sum_{j}^{\prime}\left[t_{0 j}^{\nu} b_{0 \nu}^{*}(\tau) \phi_{j \nu}(\tau)\right. \\
& \left.+t_{j 0}^{\nu} b_{0 \nu}(\tau) \phi_{\nu j}^{*}(\tau)\right] .
\end{aligned}
$$

Similarly, we define connected correlation functions for the one-particle excitations above the condensate as

$$
\begin{aligned}
& G_{j k \nu}^{11(0)}\left(\tau_{1}-\tau_{2}\right)=-\left\langle T_{\tau} b_{j \nu}\left(\tau_{1}\right) b_{k \nu}^{*}\left(\tau_{2}\right)\right\rangle_{S^{(0)}}, \\
& G_{j k v}^{22(0)}\left(\tau_{1}-\tau_{2}\right)=-\left\langle T_{r} b_{j \nu}^{*}\left(\tau_{1}\right) b_{k \nu}\left(\tau_{2}\right)\right\rangle_{S^{(0)}}, \\
& G_{j k \nu}^{12(0)}\left(\tau_{1}-\tau_{2}\right)=-\left\langle T_{\tau} b_{j \nu}\left(\tau_{1}\right) b_{k \nu}\left(\tau_{2}\right)\right\rangle_{S^{(0)}}, \\
& G_{j k \nu}^{21(0)}\left(\tau_{1}-\tau_{2}\right)=-\left\langle T_{r} b_{j \nu}^{*}\left(\tau_{1}\right) b_{k \nu}^{*}\left(\tau_{2}\right)\right\rangle_{S^{(0)}},
\end{aligned}
$$

which permits us to express the second-order contribution [Eq. (A9)] as

$$
\begin{aligned}
\frac{1}{2 !} \int_{0}^{\beta} d \tau_{1} \int_{0}^{\beta} d \tau_{2}\left\langle\Delta S\left(\tau_{1}\right) \Delta S\left(\tau_{2}\right)\right\rangle_{S^{(0)}=} & -\frac{1}{2 !} \int_{0}^{\beta} d \tau_{1} \int_{0}^{\beta} d \tau_{2} \sum_{\nu} \sum_{j k}^{\prime}\left[t_{j 0}^{\nu} t_{k 0}^{\nu} G_{j k \nu}^{21(0)}\left(\tau_{1}-\tau_{2}\right) b_{0 \nu}\left(\tau_{1}\right) b_{0 \nu}\left(\tau_{2}\right)\right. \\
& +t_{j 0}^{\nu} t_{0 k}^{\nu} G_{j k \nu}^{22(0)}\left(\tau_{1}-\tau_{2}\right) b_{0 \nu}\left(\tau_{1}\right) b_{0 \nu}^{*}\left(\tau_{2}\right)+t_{0 j}^{\nu} t_{k 0}^{\nu} G_{j k \nu}^{11(0)}\left(\tau_{1}-\tau_{2}\right) b_{0 \nu}^{*}\left(\tau_{1}\right) b_{0 \nu}\left(\tau_{2}\right) \\
& +t_{0 j}^{\nu} t_{0 k}^{\nu} G_{j k \nu}^{12(0)}\left(\tau_{1}-\tau_{2}\right) b_{0 \nu}^{*}\left(\tau_{1}\right) b_{0 \nu}^{*}\left(\tau_{2}\right)-t_{j 0}^{\nu} t_{k 0}^{\nu} \phi_{j \nu}^{*}\left(\tau_{1}\right) \phi_{k \nu}^{*}\left(\tau_{2}\right) b_{0 \nu}\left(\tau_{1}\right) b_{0 \nu}\left(\tau_{2}\right) \\
& -t_{j 0}^{\nu} t_{0 k}^{\nu} \phi_{j \nu}^{*}\left(\tau_{1}\right) \phi_{k \nu}\left(\tau_{2}\right) b_{0 \nu}\left(\tau_{1}\right) b_{0 \nu}^{*}\left(\tau_{2}\right)-t_{0 j}^{\nu} t_{k 0}^{\nu} \phi_{j \nu}\left(\tau_{1}\right) \phi_{k \nu}^{*}\left(\tau_{2}\right) b_{0 \nu}^{*}\left(\tau_{1}\right) b_{0 \nu}\left(\tau_{2}\right)
\end{aligned}
$$




$$
\left.-t_{0 j}^{\nu} t_{0 k}^{\nu} \phi_{j \nu}\left(\tau_{1}\right) \phi_{k \nu}\left(\tau_{2}\right) b_{0 \nu}^{*}\left(\tau_{1}\right) b_{0 \nu}^{*}\left(\tau_{2}\right)\right]
$$

Here, the first four terms are due to connected contributions and the last four terms due to disconnected contributions; higher terms can be written in a similar way.

A nontrivial limit $d \rightarrow \infty$ is obtained by scaling the hopping amplitudes $t_{i j}^{\nu}$ of $\nu$ bosons, as described in Sec. II B. Namely, integer scaling is applied if $t_{i j}^{\nu}$ appears together with at least one anomalous average $\phi_{i v}(\tau)=\left\langle b_{i \nu}(\tau)\right\rangle_{S^{(0)}}$ involving the BEC, while fractional scaling is employed otherwise. For example, in the first-order term [Eq. (A8)], the sum over $j$ gives a contribution of the order $O\left(Z^{R_{0 j}}\right)$ so that the hopping amplitude $t_{0 j}^{\nu}$ must be scaled with (i.e., divided by) a factor $Z^{R_{0 j}}$ because $\phi_{j \nu}$ does not depend on the distance. On the other hand, in the first four terms of the second-order contribution to the partition function [Eq. (A9)], the hopping amplitudes must be scaled with $Z^{R_{0 j} / 2}$ because the one-particle correlation functions are already proportional to $1 / Z^{R_{0 j} / 2}$, as discussed in the Sec. II B. In the last four terms of the second-order contribution, the hopping amplitudes must be scaled with $Z^{R_{0 j}}$. In the calculation of higher-order terms, one has to distinguish the cases where all site indices are different from those where some, or all, are the same. In analogy to the fermionic case, discussed in detail in Ref. 28, we find that all connected higher-order terms vanish at least as $O(1 / Z)$. Consequently, in the $Z \rightarrow \infty$ limit, only connected contributions containing $\phi_{j v}$ or $G_{j k v}^{a b(0)}$ or disconnected contributions made of products of connected contributions remain, provided the infinite series converges at least conditionally. Finally, we assume that the system is homogeneous, i.e., that $\phi_{i \nu}=\phi_{\nu}$ is site independent. Applying the linked cluster theorem and collecting only connected contributions in the exponential function, one obtains the local action

$$
\begin{aligned}
S_{\mathrm{loc}}= & \int_{0}^{\beta} d \tau b_{0 \nu}^{*}(\tau)\left(\partial_{\tau}-\mu\right) b_{0 \nu}(\tau)+\int_{0}^{\beta} d \tau \sum_{\nu} \kappa_{\nu}\left[b_{0 \nu}^{*}(\tau) \phi_{\nu}(\tau)+b_{0 \nu}(\tau) \phi_{\nu}^{*}(\tau)\right] \\
& -\int_{0}^{\beta} d \tau_{1} \int_{0}^{\beta} d \tau_{2} \sum_{\nu} \sum_{j k}^{\prime}\left[\widetilde{t}_{j 0}^{\nu} \widetilde{t}_{k 0}^{v} G_{j k \nu}^{21(0)}\left(\tau_{1}-\tau_{2}\right) b_{0 \nu}\left(\tau_{1}\right) b_{0 \nu}\left(\tau_{2}\right)+\widetilde{t}_{j 0}^{\nu} \widetilde{t}_{0 k}^{\nu} G_{j k \nu}^{22(0)}\left(\tau_{1}-\tau_{2}\right) b_{0 \nu}\left(\tau_{1}\right) b_{0 \nu}^{*}\left(\tau_{2}\right)\right. \\
& \left.+\widetilde{t}_{0 j}^{v} \tau_{k 0}^{v} G_{j k \nu}^{11(0)}\left(\tau_{1}-\tau_{2}\right) b_{0 \nu}^{*}\left(\tau_{1}\right) b_{0 \nu}\left(\tau_{2}\right)+\widetilde{t}_{0 j}^{\nu} \widetilde{t}_{0 k}^{\nu} G_{j k \nu}^{12(0)}\left(\tau_{1}-\tau_{2}\right) b_{0 \nu}^{*}\left(\tau_{1}\right) b_{0 \nu}^{*}\left(\tau_{2}\right)\right]+\frac{1}{2} \sum_{\mu \nu} U_{\mu \nu} n_{0 \mu}\left(n_{0 \nu}-\delta_{\mu \nu}\right),
\end{aligned}
$$

where the numerical factor $\kappa_{\nu}=\sum_{i \neq 0} \widetilde{t}_{i 0}^{v} / Z^{R_{i 0}}$ for $d \rightarrow \infty$ depends on the lattice structure.

To simplify notations, we introduce the Nambu formalism ${ }^{54}$ by defining a spinor boson operators $\mathbf{b}_{i \nu}$ $=\left(b_{i v}, b_{i, v}^{\dagger}\right)$ and corresponding complex variables in the pathintegral representation. Thereby, anomalous averages for the condensate

$$
\boldsymbol{\Phi}_{i \nu}(\tau) \equiv\left\langle\mathbf{b}_{i \nu}(\tau)\right\rangle_{S^{(0)}}
$$

and connected propagators for normal bosons

$$
\mathbf{G}_{i j \nu}^{(0)}\left(\tau-\tau^{\prime}\right) \equiv-\left\langle T_{\tau} \mathbf{b}_{i \nu}(\tau) \mathbf{b}_{j \nu}^{\dagger}\left(\tau^{\prime}\right)\right\rangle_{S^{(0)}}
$$

can be written in a compact vector or matrix form. Introducing the hybridization matrix function,

$$
\boldsymbol{\Delta}_{\nu}\left(\tau-\tau^{\prime}\right)=-\sum_{i j}{ }^{\prime} \widetilde{t}_{i 0}^{v} \widetilde{t}_{j 0}^{v} \mathbf{G}_{i j \nu}^{(0)}\left(\tau-\tau^{\prime}\right),
$$

and employing the free (Weiss) mean-field propagator $\mathcal{G}_{\nu}$, one can express the B-DMFT local action in the form of Eq. (10). Here, the site index $i=0$ is omitted for simplicity.
Finally, the lattice self-consistency condition [Eq. (12)] needs to be derived. For this, we apply the relation between the Green's function $\mathbf{G}_{i j \nu}^{(0)}\left(\tau-\tau^{\prime}\right)$, where the site $i=0$ is removed, and the full lattice Green's function, i.e.,

$$
\mathbf{G}_{i j \nu}^{(0)}=\mathbf{G}_{i j \nu}-\mathbf{G}_{i 0 \nu} \mathbf{G}_{00 \nu}^{-1} \mathbf{G}_{0 j \nu}
$$

which holds for a general lattice. In the B-DMFT selfconsistency [Eqs. (9)-(13)] for a homogeneous system, only the site index $i=0$ enters which is therefore dropped.

\section{APPENDIX B: FREE BOSONS ON THE BETHE TREE WITH INFINITE COORDINATION NUMBER}

In this appendix, we employ the B-DMFT to study a single species $(\nu=1)$ of noninteracting bosons on the Bethe lattice with $Z=\infty .{ }^{55,56}$ Although this problem is exactly solvable by different methods, ${ }^{57}$ it is instructive to see how the B-DMFT works in detail in this case. 


\section{Green's function method}

In order to obtain the Matsubara Green's function,

$$
G_{i j}\left(\tau-\tau^{\prime}\right)=-\left\langle T_{\tau} b_{i}(\tau) b_{j}^{\dagger}\left(\tau^{\prime}\right)\right\rangle
$$

for noninteracting bosons described by Hamiltonian (4), we use the Bogoliubov transformation to separate the operator $b_{i}$ into a normal (noncondensate) part $\tilde{b}_{i}$ and the condensate wave function $\phi_{i}$ as

$$
\begin{aligned}
& b_{i}=\tilde{b}_{i}+\phi_{i}, \\
& b_{i}^{\dagger}=\tilde{b}_{i}^{\dagger}+\phi_{i}^{*} .
\end{aligned}
$$

Assuming the system to be homogeneous, $\phi_{i}=\phi$, this yields

$$
G_{i j}\left(\tau-\tau^{\prime}\right)=-|\phi|^{2}+\widetilde{G}_{i j}\left(\tau-\tau^{\prime}\right)
$$

where $\widetilde{G}_{i j}$ is the Green's function of the normal bosons. In the noninteracting case considered here, the anomalous Green's function is absent. The density of particles is given by

$$
n=-\lim _{\tau^{\prime} \rightarrow \tau^{+}} \frac{1}{N_{L}} \sum_{i} G_{i i}\left(\tau-\tau^{\prime}\right)=|\phi|^{2}-\lim _{\tau^{\prime} \rightarrow \tau^{+}} \frac{1}{N_{L}} \sum_{i} \widetilde{G}_{i i}\left(\tau-\tau^{\prime}\right),
$$

where $N_{L}$ is the number of lattice sites. by

The diagonal Green's function of normal bosons is given

$$
\widetilde{G}_{i i}\left(\tau-\tau^{\prime}\right)=\frac{1}{\beta} \sum_{n} e^{-i \omega_{n}\left(\tau-\tau^{\prime}\right)} \widetilde{G}_{i i}\left(\omega_{n}\right)
$$

where

$$
\tilde{G}_{i i}\left(\omega_{n}\right)=\frac{1}{N_{L}} \sum_{\lambda} \frac{1}{i \omega_{n}+\mu-\lambda}=\frac{1}{i \omega_{n}+\mu-\Delta\left(\omega_{n}\right)},
$$

and $\lambda$ are the exact energy eigenstates of the lattice Hamiltonian. The recursion relation

$$
\widetilde{G}_{i i}\left(\omega_{n}\right)=\frac{1}{i \omega_{n}+\mu-\widetilde{t}^{2} \widetilde{G}_{i i}\left(\omega_{n}\right)},
$$

which is exact for the Bethe lattice, ${ }^{55,56}$ allows one to express the hybridization function as

$$
\Delta\left(\omega_{n}\right)=\widetilde{t}^{2} \widetilde{G}_{i i}\left(\omega_{n}\right)
$$

Equation (B7) determines $\widetilde{G}_{i i}$ as

$$
\tilde{G}_{i i}\left(\omega_{n}\right)=\frac{i \omega_{n}+\mu-\sqrt{\left(i \omega_{n}+\mu\right)^{2}-4 \widetilde{t}^{2}}}{2 \tilde{t}^{2}} .
$$

In particular, the equation for the particle density follows as

$$
n=|\phi|^{2}-\frac{2}{\beta} \sum_{n} \frac{e^{i \omega_{n} 0^{+}}}{i \omega_{n}+\mu+\sqrt{\left(i \omega_{n}+\mu\right)^{2}-4 \widetilde{t}^{2}}} .
$$

Cauchy's theorem allows one to express the infinite sum as an integral over the spectral function multiplied by the BoseEinstein distribution function ${ }^{36,53,54}$ such that the density equation takes the form

$$
n=|\phi|^{2}+\frac{1}{2 \pi \tilde{t}^{2}} \int_{-2 \widetilde{t}}^{-2 \widetilde{t}} d \omega \frac{\sqrt{4 \widetilde{t}^{2}-\omega^{2}}}{e^{\beta(\omega-\mu)}-1} .
$$

For temperatures $T>T_{\mathrm{BEC}}$, the condensate vanishes, $|\phi|=0$, in which case the equation determines $\mu$ as a function of the density $n$. For $T<T_{\mathrm{BEC}}$, the chemical potential is pinned at the value $\mu=-2 \widetilde{t}$ and Eq. (B11) determines $|\phi|^{2}$, the density of the condensate. The condensation temperature $T_{\mathrm{BEC}}$ itself is thus obtained for $\mu=-2 \tilde{t}$ and $|\phi|=0$. Expanding the BoseEinstein function into a Taylor series and changing the integration variable into $\omega=2 \widetilde{t} \cos \theta$, one obtains a transcendental equation for $T_{\mathrm{BEC}}$,

$$
n=\frac{4 \pi \vec{t}^{2}}{x} \sum_{k=1}^{\infty} \frac{e^{-k x}}{k} I_{1}(k x),
$$

where $x=2 \widetilde{t} / T_{\mathrm{BEC}}$ and $I_{1}$ is a modified Bessel function. ${ }^{58}$

\section{Bosonic dynamical mean-field theory}

We now show that the same results can be derived directly from the B-DMFT equations. The local action takes the explicit form

$$
\begin{aligned}
S_{\mathrm{loc}}= & -\int_{0}^{\beta} d \tau \int_{0}^{\beta} d \tau^{\prime} b^{*}(\tau) \mathcal{G}^{-1}\left(\tau-\tau^{\prime}\right) b\left(\tau^{\prime}\right) \\
& +\tilde{t} \int_{0}^{\beta} d \tau\left[b^{*}(\tau) \phi(\tau)+\phi^{*}(\tau) b(\tau)\right],
\end{aligned}
$$

where the local Weiss Green,s function (an operator) is given by

$$
\mathcal{G}^{-1}\left(\tau-\tau^{\prime}\right)=\delta\left(\tau-\tau^{\prime}\right)\left(-\partial_{\tau}+\mu\right)-\Delta\left(\tau-\tau^{\prime}\right) .
$$

The hybridization function $\Delta(\tau)$ is determined selfconsistently by Eqs. (11) and (12). As in the fermionic case, the relation $\Delta\left(\omega_{n}\right)=\widetilde{t}^{2} \mathcal{G}\left(\omega_{n}\right)$ also holds for noninteracting bosons on the Bethe lattice in the limit $Z \rightarrow \infty$.

In the absence of interactions, the Euler-Lagrange equation of motion for the classical field $\phi(\tau)$ is given by

$$
\begin{aligned}
0=\left.\frac{\delta S_{\mathrm{loc}}\left[b, b^{*}\right]}{\delta b^{*}(\tau)}\right|_{b(\tau)=\phi(\tau)}= & \left(\partial_{\tau}-\mu\right) \phi(\tau) \\
& +\int_{0}^{\beta} d \tau^{\prime} \Delta\left(\tau-\tau^{\prime}\right) \phi\left(\tau^{\prime}\right)+\widetilde{t} \phi(\tau) .
\end{aligned}
$$

By Fourier transformation, Eq. (B15) becomes a linear equation,

$$
\left[i \omega_{n}+\mu-\tilde{t}-\Delta\left(\omega_{n}\right)\right] \phi\left(\omega_{n}\right)=0 .
$$

Employing Eqs. (B9) and (B10), this equation takes the form 


$$
\left[i \omega_{n}+\mu-2 \widetilde{t}+\sqrt{\left(i \omega_{n}+\mu\right)^{2}-(2 \widetilde{t})^{2}}\right] \phi\left(\omega_{n}\right)=0 .
$$

In the static limit, $\omega_{n}=0$, corresponding to $n=0$, this equation has the solution $\phi=0$ when $\mu<-2 \widetilde{t}$, implying that the chemical potential lies outside the bosonic band, or the solution $\phi \neq 0$ when $\mu=-2 \widetilde{t}$. In the latter case, the actual value of $|\phi|$ (which determines the BEC fraction) must be computed from the equation for the particle density [Eq. (B11)]. In the dynamical case, $\omega_{n} \neq 0$, i.e., for $n \neq 0$, Eq. (B17) only has the solution $\phi\left(\omega_{n}\right)=0$ because the expression in the bracket never vanishes. This shows that for noninteracting bosons, the condensate order parameter is time independent.

After Fourier transformation, the local action takes the form

$$
S_{\mathrm{loc}}=\sum_{n} b_{n}^{*}\left[i \omega_{n}+\mu-\Delta\left(\omega_{n}\right)\right] b_{n}+\widetilde{t}\left(b_{n=0}^{*} \phi+b_{n=0} \phi^{*}\right),
$$

where the numbers $b_{n}$ are the Fourier coefficients of $b(\tau)$ in the Matsubara frequency space. The zero frequency component $b_{n=0}$ is shifted by the Bogoliubov transformation [Eq. (B2)] as $b_{n=0}=\widetilde{b}_{n=0}+\phi$. Because of Eq. (B16), the local action is seen to be quadratic in $\widetilde{b}_{n}$ and the functional integral yields the same equation for the particle density as Eq. (B11). Thus, we showed that the B-DMFT correctly reproduces all results for noninteracting bosons on the Bethe lattice both in the normal and in the BEC phase.
${ }^{1}$ R. J. Anglin and W. Ketterle, Nature (London) 416, 211 (2002).

${ }^{2}$ M. Greiner, O. Mandel, T. Esslinger, T. W. Hänsch, and I. Bloch, Nature (London) 415, 39 (2002).

${ }^{3}$ M. Lewenstein, A. Sanpera, V. Ahufinger, B. Damski, A. Sen, and U. Sen, Adv. Phys. 56, 243 (2007).

${ }^{4}$ I. Bloch, J. Dalibard, and W. Zwerger, arXiv:0704.3011, Rev. Mod. Phys. (to be published).

${ }^{5}$ D. Jaksch, C. Bruder, J. I. Cirac, C. W. Gardiner, and P. Zoller, Phys. Rev. Lett. 81, 3108 (1998).

${ }^{6}$ A. Isacsson, M.-Ch. Cha, K. Sengupta, and S. M. Girvin, Phys. Rev. B 72, 184507 (2005).

${ }^{7}$ S. D. Huber, E. Altman, H. P. Büchler, and G. Blatter, Phys. Rev. B 75, 085106 (2007).

${ }^{8}$ S. Sachdev, K. Sengupta, and S. M. Girvin, Phys. Rev. B 66, 075128 (2002).

${ }^{9}$ A. Micheli, G. K. Brennen, and P. Zoller, Nat. Phys. 2, 341 (2006).

${ }^{10}$ T. Matsubara and H. Matsuda, Prog. Theor. Phys. 16, 569 (1956).

${ }^{11}$ T. Matsubara and H. Matsuda, Prog. Theor. Phys. 17, 19 (1957).

${ }^{12}$ T. Morita, Prog. Theor. Phys. 18, 462 (1957).

${ }^{13}$ M. P. A. Fisher, P. B. Weichman, G. Grinstein, and D. S. Fisher, Phys. Rev. B 40, 546 (1989).

${ }^{14}$ G. G. Batrouni, R. T. Scalettar, and G. T. Zimanyi, Phys. Rev. Lett. 65, 1765 (1990).

${ }^{15}$ D. S. Rokhsar and B. G. Kotliar, Phys. Rev. B 44, 10328 (1991).

${ }^{16}$ K. Sheshadri, H. R. Krishnamurthy, R. Pandit, and T. V. Ramakrishnan, Europhys. Lett. 22, 257 (1993).

${ }^{17}$ J. K. Freericks and H. Monien, Europhys. Lett. 26, 545 (1994).

${ }^{18}$ J. K. Freericks and H. Monien, Phys. Rev. B 53, 2691 (1996).

${ }^{19}$ J. D. Reppy, J. Low Temp. Phys. 87, 205 (1992).

${ }^{20}$ A. P. Kampf and G. T. Zimanyi, Phys. Rev. B 47, 279 (1993).

${ }^{21}$ C. Bruder, R. Fazio, and G. Schön, Ann. Phys. (Leipzig) 14, 566 (2005).

${ }^{22}$ T. Nikuni, M. Oshikawa, A. Oosawa, and H. Tanaka, Phys. Rev. Lett. 84, 5868 (2000); Ch. Rüegg, N. Cavadini, A. Furrer, H.-U. Güdel, K. Krämer, H. Mutka, A. Wildes, K. Habicht, and P. Vorderwisch, Nature (London) 423, 62 (2003).

${ }^{23}$ T. Giamarchi, Ch. Rüegg, and O. Tchernyshyov, Nat. Phys. 4, 198 (2008).

${ }^{24}$ E. Kim and M. H. W. Chan, Nature (London) 427, 225 (2004).
${ }^{25}$ A. J. Leggett, Quantum Liquids (Oxford University Press, New York, 2006).

${ }^{26}$ W. Metzner and D. Vollhardt, Phys. Rev. Lett. 62, 324 (1989).

${ }^{27}$ Th. Pruschke, M. Jarrell, and J. K. Freericks, Adv. Phys. 44, 187 (1995).

${ }^{28}$ A. Georges, G. Kotliar, W. Krauth, and M. J. Rozenberg, Rev. Mod. Phys. 68, 13 (1996).

${ }^{29}$ G. Kotliar and D. Vollhardt, Phys. Today 57(3), 53 (2004).

${ }^{30}$ H. A. Gersch and G. C. Knollman, Phys. Rev. 129, 959 (1963).

${ }^{31}$ G. Kotliar, S. Y. Savrasov, K. Haule, V. S. Oudovenko, O. Parcollet, and C. A. Marianetti, Rev. Mod. Phys. 78, 865 (2006).

${ }^{32}$ C. Itzykson and J.-M. Drouffe, Statistical Field Theory (Cambridge University Press, Cambridge, 1989), Vols. I and II.

${ }^{33}$ E. Müller-Hartmann, Z. Phys. B: Condens. Matter 74, 507 (1989).

${ }^{34}$ Q. Si and J. L. Smith, Phys. Rev. Lett. 77, 3391 (1996).

${ }^{35}$ H. Shi and A. Griffin, Phys. Rep. 304, 1 (1998).

${ }^{36}$ A. A. Abrikosov, L. P. Gorkov, and I. E. Dzyaloshinski, Methods of Quantum Field Theory in Statistical Physics (Dover, New York, 1963).

${ }^{37}$ L. Erdos, B. Schlein, and H.-T. Yau, Commun. Pure Appl. Math. 59, 1659 (2006).

${ }^{38}$ L. Erdos, B. Schlein, and H.-T. Yau, Phys. Rev. Lett. 98, 040404 (2007).

${ }^{39}$ D. H. Kobe, Phys. Rev. A 5, 854 (1972).

${ }^{40}$ S. Stenholm, Phys. Rev. A 57, 2942 (1998).

${ }^{41}$ J.-B. Bru and T. C. Dorlas, J. Stat. Phys. 113, 177 (2003).

${ }^{42}$ P. van Dongen and D. Vollhardt, Phys. Rev. B 40, 7252 (1989).

${ }^{43}$ W. Krauth, M. Caffarel, and J.-Ph. Bouchaud, Phys. Rev. B 45, 3137 (1992).

${ }^{44}$ S. Sachdev, Quantum Phase Transitions (Cambridge University Press, Cambridge, England, 1999).

${ }^{45}$ E. Altman, W. Hofstetter, E. Demler, and M. D. Lukin, New J. Phys. 5, 113 (2003).

${ }^{46}$ I. Titvinidze, M. Snoek, and W. Hofstetter, Phys. Rev. Lett. 100, 100401 (2008).

${ }^{47}$ J. K. Freericks and V. Zlatić, Rev. Mod. Phys. 75, 1333 (2003).

${ }^{48}$ C. Ates and K. Ziegler, Phys. Rev. A. 71, 063610 (2005).

${ }^{49}$ M. M. Maska, R. Lemanski, J. K. Freericks, and C. J. Williams, arXiv:0802.3894 (unpublished).

${ }^{50}$ P. A. Martin and J. Piasecki, Phys. Rev. E 68, 016113 (2003); 
71, 016109 (2005).

${ }^{51}$ A quantum-mechanical system of noninteracting particles, which is coupled to a single site ("impurity"), where interactions are possible defines a so-called quantum-mechanical "singleimpurity problem." In particular, the "bosonic single-impurity problem" discussed here requires the calculation of the local Green's function [Eq. (9)] with an arbitrary local action [Eq. (10)].

${ }^{52}$ H.-J. Lee and R. Bulla, Eur. Phys. J. B 56, 199 (2007).

${ }^{53}$ J. W. Negele and H. Orland, Quantum Many-Particle Systems (Addison-Wesley, Menlo Park, 1988).

${ }^{54} \mathrm{G}$. Rickayzen, Green's Functions and Condensed Matter (Aca- demic, London, 1984).

${ }^{55}$ M. Eckstein, M. Kollar, K. Byczuk, and D. Vollhardt, Phys. Rev. B 71, 235119 (2005).

${ }^{56}$ M. Kollar, M. Eckstein, K. Byczuk, N. Blümer, P. van Dongen, M. H. Radke de Cuba, W. Metzner, D. Tanaskovic, V. Dobrosavljevic, G. Kotliar, and D. Vollhardt, Ann. Phys. (Leipzig) 14, 642 (2005).

${ }^{57}$ M. van den Berg, T. C. Dorlas, and V. B. Priezzhev, J. Stat. Phys. 69, 307 (1992).

${ }^{58}$ M. Abramowitz and I. A. Stegun, Handbook of Mathematical Functions (Dover, New York, 1972). 